\title{
Hypodiploid B Acute Lymphoblastic Leukemia
}

National Cancer Institute

\section{Source}

National Cancer Institute. Hypodiploid B Acute Lymphoblastic Leukemia. NCI Thesaurus.

Code C80345.

A B-cell acute leukemia characterized by the presence of lymphoblasts which contain less than 46 chromosomes. It occurs in both children and adults. It has an unfavorable clinical outcome. 